\title{
Convergence Properties of an Online Learning Algorithm in Neural Network Models of Complex Systems
}

\author{
V.N. Azarskov, S.A. Nikolaienko \\ Aircraft Control Systems Dept. \\ National Aviation University \\ Kyiv, Ukraine \\ azarskov@nau.edu.ua \\ s_nicolaenko@ukr.net
}

\author{
L.S. Zhiteckii \\ Int. Centre of Inform. Tech. \& Syst. \\ Institute of Cybernetics \\ Kyiv, Ukraine \\ leonid_zhiteckii@i.ua
}

\section{Problem Formulation}

Let

$$
y=F(x)
$$

be some nonlinear unknown function describing a complex system. In this equation, $y \in \operatorname{IR}$ and $x \in \mathrm{IR}^{N}$ are the output scalar and input vector variables, respectively, available for the measurement at each nth time instant $(n=1,2, \ldots)$. This implies that

$$
y(n)=F(x(n-1))
$$

with an unknown mapping $F: \mathrm{IR}^{N} \rightarrow \mathrm{IR}$.

To approximate (1), the two-layer neural network containing $M(M \geq 1)$ neurons in its hidden layer is employed. The inputs to the each jth neuron of this layer at the time instant $n$ are the components of $x(n-1)$. Its output signal at the nth time instant is given by

$$
y_{j}^{(1)}(n)=\sigma\left(b_{j}^{(1)}+\sum_{i=1}^{N} w_{i j}^{(1)} x_{i}(n-1)\right), \quad j=1, \ldots, M,
$$

where $x_{i}(n-1)$ denotes the ith component of $x(n-1)$, and $w_{i j}^{(1)}$ and $b_{j}^{(1)}$ are the weight coefficients and the bias of this $\mathrm{jth}$ neuron, respectively. $\sigma(\cdot)$ represents the so-called activation function. There is only one neuron in the output (second) layer, whose inputs are the outputs of the hidden layer's neurons. The output signal of second layer, $y^{(2)}(n)$, at the time instant $n$ is determined by 


$$
y^{(2)}(n)=\sum_{j=1}^{M} w_{j}^{(2)} y_{j}^{(1)}(n)+b^{(2)}
$$

where $w_{1}^{(2)}, \ldots, w_{M}^{(2)}$ are the weights of this neuron and $b^{(2)}$ is its bias.

Since $\sigma(\cdot)$ is assumed to be nonlinear, it follows from (3), (4) together with (2) that $y^{(2)}(n)$ is a nonlinear function depending on $x(n-1)$ and also on the $(M(N+2)+1)$ dimensional parameter vector

$$
w=\left[w_{11}^{(1)}, \ldots, w_{N 1}^{(1)}, b_{1}^{(1)}, \ldots, w_{1 M}^{(1)}, \ldots, w_{N M}^{(1)}, b_{M}^{(1)} \vdots w_{1}^{(2)}, \ldots, w_{M}^{(2)}, b^{(2)}\right]^{T} .
$$

To emphasize this fact, define the output signal of the neural network in the form

$$
y^{(2)}(n)=\mathrm{NN}(x(n-1), w)
$$

with $\mathrm{NN}: \mathrm{IR}^{N} \times \mathrm{IR}^{M(N+2)+1} \rightarrow \mathrm{IR}$.

The following basic assumption is made. There exists at least an unique $w=w^{*} \in \mathrm{IR}^{M(N+2)+1}$ such that $F(x)$ can explicitly be approximated by $\mathrm{NN}\left(x, w^{*}\right)$, in the sense of

$$
F(x) \equiv \mathrm{NN}\left(x, w^{*}\right)
$$

for all $x$ from a given compact set $X \subset \mathrm{IR}^{N}$.

Define the training sequence $\{(x(n-1), y(n))\}_{n=1}^{\infty}$ of the measurable pairs in which $x(n-1) \mathrm{s}$ are taken randomly from $X$. Then, the online learning algorithm for updating the parameter estimate $w(n)$ is formulated as the following standard recursive gradient procedure:

$$
w(n)=w(n-1)+\eta \widetilde{e}(n, w(n-1)) \operatorname{grad}_{w} \mathrm{NN}(x(n-1), w(n-1)) .
$$

In this algorithm,

$$
\widetilde{e}(n, w(n-1))=y(n)-\mathrm{NN}(x(n-1), w(n-1))
$$

is the current estimation error and $\operatorname{grad}_{w} \mathrm{NN}(x(n-1), w(n-1))$ denotes the gradient of $\mathrm{NN}(x, w)$ at the point $w=w(n-1)$, and $\eta \equiv$ const $>0$ is its step size (the learning rate).

The problem is to study the properties of sequence $\{w(n)\}$ caused by (7), (8) as $n$ tends to $\infty$.

Equations (2) and (7) together with (5), (6) and (8) describe the closed-loop system for adaptive identification of (1).

\section{Convergence Analysis}

\section{A. Preliminaries}

To analyze the asymptotic behavior of (7), (8), the scalar non-negative function $V(w)$ given by

$$
V(w)=0 \text { for } w=w^{*}, \quad V(w)>0 \text { for } w \neq w^{*}
$$

is exploited.

The variable $V_{n}:=V(w(n))$ becomes immediately the Lyapunov function of the algorithm (7), (8) if only

$$
V_{n} \leq V_{n-1} \quad \forall n .
$$

Since $V_{n} \geq 0$, the condition (10) under which $V_{n}$ does not increase is sufficient for existing a limit

$$
\lim _{n \rightarrow \infty} V_{n}=V_{\infty}
$$

where $V_{\infty}$ is a random number depending on $w(0)$ and $\{x(n)\}$.

In the presence of the one-point $W^{*}=\left\{w^{*}\right\}$, the function $V(w)$ satisfying $(9)$ is usually chosen as

$$
V(w)=\left\|w^{*}-w\right\|^{2},
$$

where \|\|$\|$ denotes the Euclidean vector norm. It turned out that if the neural network contains the hidden layer, then $W^{*}$ consists of several isolated $w^{*} \mathrm{~s}$. In particular, in the simplest case, when there is one neuron in the hidden layer $(N=1, M=1)$ and $\sigma(\cdot)$ is described by

$$
\sigma(s)=\frac{1}{1+\exp (-s)},
$$

the set $W^{*}$ contains two points: $\mathbf{w}_{1}^{*}=\left[w_{1}^{*}, w_{2}^{*}, w_{3}^{*}, w_{4}^{*}\right]^{T}$ and $\mathbf{w}_{2}^{*}=\left[-w_{1}^{*},-w_{2}^{*},-w_{3}^{*}, w_{3}^{*}+w_{4}^{*}\right]^{T}$.

In the case when $W^{*}$ is not one-point, $V(w)$ is designed as

$$
V(w)=\inf _{w^{*} \in W^{*}}\left\|w^{*}-w\right\|^{2}
$$

but not as defined in (12).

We first observed in simulation examples that $\{w(n)\}$ may not converge even in the presence of bounded $\{x(n)\}$ if there are no additional restrictions on this input sequence. Such an ultimate feature of (7), (8) implies that 


$$
\lim _{n \rightarrow \infty} w(n)=w_{\infty}
$$

may not exist. Nevertheless, if (15) is achieved, then the following asymptotic properties of $\{w(n)\}$ can be established:

a) $\{w(n)\}$ converges to some $w_{\infty}$ in sense of (15) with

$$
w_{\infty} \in \lim \inf W_{n}
$$

where

$$
\lim \inf W_{n}:=\bigcup_{n=1}^{\infty} \bigcap_{k=n}^{\infty} W_{k}
$$

denotes the limit set in which

$$
W_{n}:=\{w: y(n)-\mathrm{NN}(x(n-1), w)=0\}
$$

b) the identification error given by (8) satisfies

$$
\lim _{n \rightarrow \infty} \widetilde{e}(n, w(n-1))=0 .
$$

Note that $\lim \inf W_{n}$ represents a nonlinear manifold on $\mathbb{R}^{M(N+2)+1}$ whose dimension satisfies

$$
0 \leq \operatorname{dim} \lim \inf W_{n} \leq M(N+2)
$$

\section{B. Simulation Examples}

To demonstrate these asymptotic properties, three simulation experiments with

$$
y=\frac{3.75+0.05 \exp (-7.15 x)}{1+0.19 \exp (-7.15 x)}
$$

was performed. This nonlinear function can explicitly be approximated by the two-layer neural network described by (5), $(6),(8)$ and (13) with $w_{11}^{(1) *}=7.15, b_{1}^{(1) *}=1.65, w_{1}^{(2) *}=3.45$, $b^{(2) *}=0.3$. In all of these experiments, $\eta$ was taken as $\eta=0.02$.

The simulation results are depicted in Figures 1-3.

Fig. 1 shows that $\left\{V_{n}\right\}$ has no limit if the input sequence $\{x(n)\}$ is non-stochastic. (The definition of the non-stochastic sequence can be found in the paper [9].) In this case, the model error $\tilde{e}(n, w(n-1))$ does not go to zero, i. e., (16) is not satisfied.
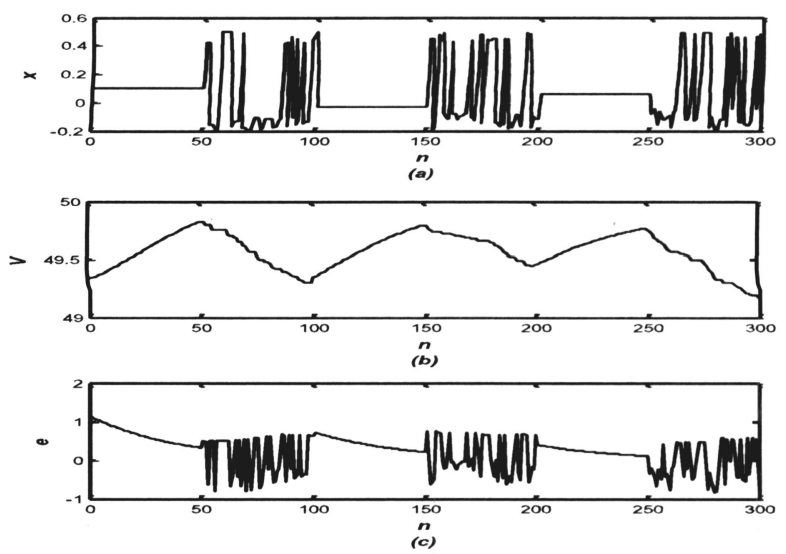

Fig. 1. Learning processes in simulation experiment 1 : (a) the input signal; (b) the function $V_{n}$ given by (14); (c) the current model error

In second experiment, $x(n) \mathrm{s}$ were sequentially chosen from the finite set containing three points: $x^{(1)}=-0.4442$; $x^{(2)}=0.5158 ; x^{(3)}=0.8761$. Fig. 2 illustrates the result of this experiment with initial $w_{11}^{(1)}(0)=0.529, \quad b_{1}^{(1)}(0)=-0.5012$, $w_{1}^{(2)}(0)=-0.9168, b^{(2)}(0)=1.0409$.

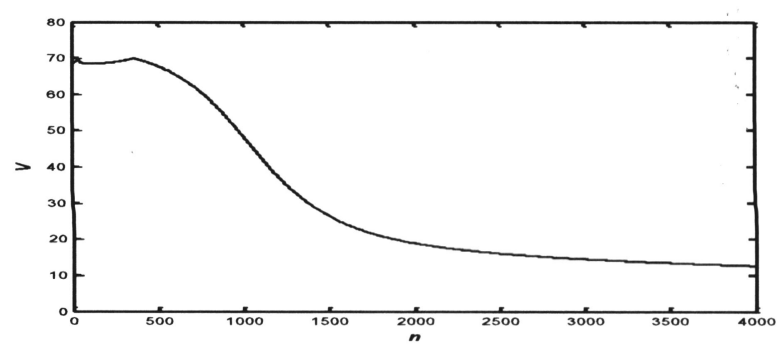

(a)

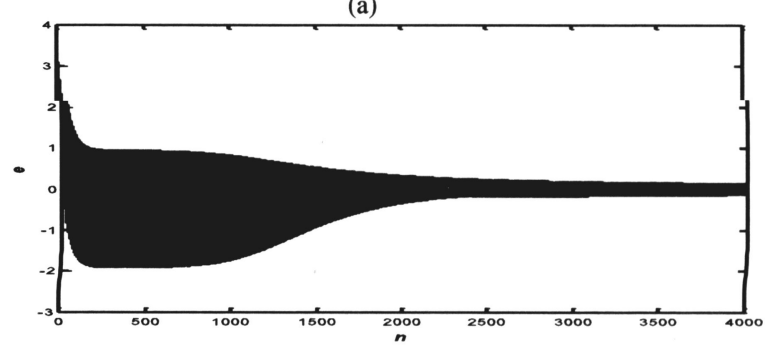

(b)

Fig. 2. Learning processes in simulation experiment 2: (a) the function $V_{n}$ given by (14); (b) the current model error

We can observe that $\left\{V_{n}\right\}$ is convergent, however its convergence is not monotonic as in (10). It turned out that in this case, $\{w(n)\}$ converges to $w_{\infty}=[5.4120,1.3172,3.8233$, $-0.0475]^{T}$ which lies on $\lim \inf W_{n}$. but not to one of two points $w_{1}^{*}=[7.15,1.65,3.45,0.3]^{T}$ or to $w_{1}^{*}=[-7.15,-1.65$, $-3.45,3.75]^{T}$. 
The case where $\left\{V_{n}\right\}$ converges monotonically is demonstrated in Fig. 3. In this case, the initial estimates were chosen as follows: $w_{11}^{(1)}(0)=1.4, \quad b_{1}^{(1)}(0)=-0.1, \quad w_{1}^{(2)}(0)=-0.56$, $b^{(2)}(0)=0.46$.

It turned out that $\{w(n)\}$ tends to the limit point $w_{1}^{*}$ $=[7.15,1.65,3.45,0.3]^{T}$ as $n \rightarrow \infty$.

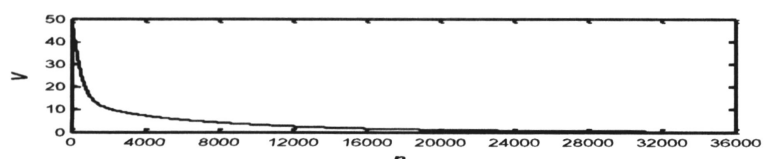

(a)

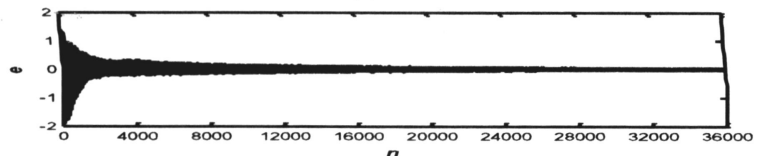

(b)

Fig. 3. Learning processes in simulation experiment 3:

(a) the function $V_{n}$ given by (14); (b) the current model error

\section{MAJN RESULT}

Main theoretical result concerning the asymptotical behavior of $(7),(8)$ is based on following additional assumptions:

$\left\{x_{i}(n)\right\}$ are the stochastic sequences of independent random variables having the probability density function

$$
p(x(n) \mid x(n-1), \ldots, x(0)) \equiv p(x(n)):=p(x)
$$

with the properties that

$$
P\left\{x(n) \in X^{\prime}\right\}=\int_{x \in X^{\prime}} p(x) d x>0
$$

for any subset $X^{\prime} \subset X$, and

$$
P\left\{x(n) \in X^{\prime \prime}\right\}=0
$$

if $\operatorname{dim} X^{\prime \prime}=0$, where $P\{\cdot\}$ denotes the probability of corresponding event.

Let $W\left(w^{*}\right)$ denote a neighborhood of some $w^{*} \in W^{*}$ which does not contain another points of $W^{*}$. With this $W\left(w^{*}\right)$, we have established that if the assumptions (6), (17) (19) are satisfied and the conditions

$$
0<\eta<2
$$

$$
\begin{aligned}
& \int_{x \in X}\left[\mathrm{NN}\left(x, w^{*}\right)-\mathrm{NN}(x, w)\right] \operatorname{grad}_{w}^{T} \mathrm{NN}(x, w)\left(w^{*}-w\right) p(x) d x \\
& \geq \int_{x \in X}\left[\mathrm{NN}\left(x, w^{*}\right)-\mathrm{NN}(x, w)\right]^{2}\left\|\operatorname{grad}_{w} \mathrm{NN}(x, w)\right\|^{2} p(x) d x
\end{aligned}
$$

hold for any $x \in X$ and for arbitrary $w$ from $W\left(w^{*}\right)$, then the limit (11) is valid with probability 1 . Again,

$$
\lim _{n \rightarrow \infty} w(n)=w^{*}
$$

almost sure (a. s.).

The proof of this result essentially utilizes the BorelCantelli lemma and Doob's martingale convergence theorem (see [9]).

\section{CONCLUSION}

In general case, the standard online gradient algorithms ap. plied to sequential learning in neural networks with hidden layer may not converge. To guarantee their convergence, certain conditions need to be satisfied.

\section{REFERENCES}

[1] L. Behera, S. Kumar, and A. Patnaik, "On adaptive learning rate th guarantees convergence in feedforward networks," IEEE Trans. on Ner ral Networks, vol. 17, pp. 1116-1125, May 2006.

[2] H. Shao, W. Wu, and L. Liu, "Convergence and monotonicity of online gradient method with penalty for neural networks," WSEA Trans. Math., vol. 6, pp. 469-476, 2007.

[3] V. Tadic and S. Stankovic, "Learning in neural networks by normalize stochastic gradient algorithm: Local convergence," in Proc. 5th Semin Neural Netw. Appl. Electr. Eng, Yugoslavia, Sept. 2000, pp. 11-17.

[4] Wu, W., G. Feng, and X. Li, "Training multilayer perceptrons via mi imization of ridge functions," Advances in Comput. Mathemati vol. 17, pp. 331-347, 2002

[5] W. Wu, G. Feng, X. Li, and Y. Xu, "Deterministic convergence of online gradient method for BP neural networks, "IEEE Trans. on Ner Networks, vol. 16, pp. 1-9, March 2005.

[6] N. Zhang, W. Wu, and G. Zheng, "Convergence of gradient method momentum for two-layer feedforward neural networks," IEEE Trans

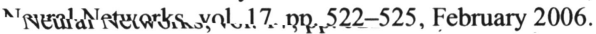

[7] H. Zhang, W. Wu, F. Liu, and M. Yao, "Boundedness and conve of online gradient method with penalty for feedforward neur works," IEEE Trans. Neural Networks, vol. 20, pp. 1050-105 2009.

[8] Z. Xu, R. Zhang, and W. Jing "When does online BP trainit verge?" IEEE Trans. Neural Networks, vol. 20, pp. 1529-1539, ber 2009 .

[9] L.S. Zhiteckii, V.N. Azarskov, and S.A. Nikolaienko, "Conver learning algorithms in neural networks for adaptive identific nonlinearly parameterized systems," in Proc. 16th IFAC Sympr System Identification, Brussels, Belgium, July $10-13,2012$, p 1598 . 